\title{
Equilibrium Assignment Model and Algorithm for Multi-Modal Considering Distance Factor
}

\author{
Zhou Hao ${ }^{1, a}$ \\ ${ }^{1}$ School of Traffic and Transportation, Beijing Jiaotong University, Beijing 100044, China \\ a15120748@bjtu.edu.cn
}

\begin{abstract}
Keywords: urban traffic; travel distance; user equilibrium; multimodal traffic super network; algorithm

Abstract: Travel distance is an important factor that influences the traffic mode choice and route choice. Considering the influences that travel distance exerts on different perceived costs, a multimodal user equilibrium assignment method is proposed. Firstly, travel behaviors of travelers in the multimodal transportation system are analyzed. Based on the graph theory, a multimodal super-network model is described, besides the effective hyper-path and the sub-path is defined. Secondly, the calculation methods of two main quantitative factors including time and cost in different travel processes are analyzed, and the generalized cost functions of travel are constructed considering the impacts that travel distance exerts on the perceived costs of travelers. On the basis of these mentioned above, a multimodal traffic network assignment model based on user equilibrium criterion is proposed, and a shortest path search algorithm based on sub-path cost is proposed. Finally, a simple example is applied to validate the model. The results show that the proposed model and algorithm are feasible and effective.
\end{abstract}

\section{Introduction}

The continuous progress of urbanization makes the inhabitant travel distance increasing, and there are more and more travel modes for travelers to choose from. Each mode has different advantages in different distance range, and travel distance is an important factor of influencing mode choice. In addition, travel distance increase will make travel cost rise correspondingly, distance factor also can affect path choice. In the multimodal transportation system, the traveler's choice behavior will directly decide its travel activities, and the gathering of travel activities forms the state of network traffic flow. Therefore, it is important for traffic management to study the travel behavior and the problem of multimodal traffic assignment considering distance factor.

Since Wardrop put forward the concept of "user equilibrium", the theory of traffic flow assignment has developed rapidly. However, most studies only carry out the single mode traffic network, such as urban transit network passenger flow assignment[1], urban rail transit network passenger flow assignment [2] and so on. At present, many scholars begin to pay attention to the problem of multimodal traffic assignment, SI, etc using hierarchical network structure to describe multimodal traffic network, and a variational inequality model considering the interferences between modes is proposed[3]. Wang, etc. build a multimodal network considering travel rules and transfer conditions[4]. And a path search algorithm based on transfer condition is proposed. With the development of new energy transport, scholars begin to pay attention to the problem of traffic flow assignment with path constraints. Kang studies the choice of the charging path of the traveler based on the activity[5]; He etc proposed the traffic flow assignment model of the distance constraint considering the single mode network of the electric vehicle[6]. In the previous researches, the generalized cost function did not consider the effect of distance factor on the traveler's choice behavior, only considering the path constraint in single mode, and did not consider the influence of the distance factor on the travel behavior.

In this paper, we study the problem of multi-mode network assignment, consider the traveler's mode selection and route selection, and analyze the effect of distance on the traveler's generalized perceived cost, and propose a multimodal equilibrium assignment model and algorithm considering the distance factor. Firstly, the travel process in the multimodal network is analyzed, and a super 
network model to describe the multimodal combined travel based on theory is established, and the effective hyper-path and sub-path are defined. Then, the travel cost of travelers in different stages is analyzed, considers the effect of distance on time and price, and the generalized cost model of multimodal combined travel is constructed. Based on this, a multimodal network assignment model based on user equilibrium and an efficient shortest path search algorithm are proposed. Finally, the model and algorithm are validated and analyzed by an example.

\section{Multimodal Hyper-network model}

In the multimodal transportation network, the traveler completes one trip often needs to experience four processes, including board, travel, transferring and leaving. According to the travel characteristics, the Super network structure is used to describe the multimodal traffic system. In multimodal hyper-network, each mode has its own traveling sub-net, different sub-net connected through the transfer node, can be described as:

$$
G=(M, V, A) \text {. }
$$

where $G$ denotes the multimodal network, $M$ denotes the set of traffic modes, $V$ denotes the set of traffic nodes, $A$ denotes the set of arcs.

In multimodal hyper-networks, nodes and arcs can be classified into different categories according to their attributes, and can be described by different state vectors. Nodes are divided into travel endpoints and non-travel endpoints, the trip endpoint represents the original or destination of the travel, where the elements are represented by a single variable $\mathrm{r}$ or $\mathrm{s}$ representation, i.e. $r, s \in V_{1}$, the non-travel endpoint represents the middle nodes of the travel process, which is represented by a set of nodes $V_{2}$, in which the elements are represented by two-dimensional vectors $(m, v)$, i.e. $(m, v) \in V_{2}$, and represents the mode and location properties of a node, respectively. In the multimodal hyper-network, arcs are divided into the boarding arcs, traveling arcs, transferring arcs and leaving arcs, different categories of arcs are represented by set $A_{1} 、 A_{2} 、 A_{3}$ and $A_{4}$, can be described as:

$$
A=A_{1} \cup A_{2} \cup A_{3} \cup A_{4} .
$$

We can use the two adjacent nodes to represent an arc, such as the boarding arc, travel arc, transfer arc and leaving arc can be expressed as $\{r,(m, v)\},\left\{\left(m, v_{1}\right),\left(m, v_{2}\right)\right\},\left\{\left(m_{1}, v\right),\left(m_{2}, v\right)\right\}$, and $\{(m, v), s\}$.

In the multimodal network, the process of the traveler's trip can be described as a sequence of points or arcs. In this paper, the concept of hyper-path is proposed to describe the travel process, which refers to a sequence of nodes connected from the original to the destination in the multimodal hyper-network, and a hyper-path can be expressed as:

$$
r-\left(m_{1}, v_{l}\right)-\left(m_{1}, v_{2}\right)-\ldots-\left(m_{1}, v_{n}\right)-\left(m_{2}, v_{n}\right)-\ldots-\left(m_{n}, v_{w}\right)-s \text {. }
$$

In practice, not all of the hyper-paths are considered, we need to confined the hyper-path, define a path that satisfies the following criteria as a effective hyper-path, and assume that the traveler only considers a valid hyper-path when choosing a trip, and that the set of valid hyper paths is recorded as $K_{r s}$ :

(1)the transfer times is no more than maximum transfer times $n_{\max }$;

(2)No continuous transfer and loop;

(3)Travel distance limit mode (such as electric car), the travel distance does not exceed the maximum value $L_{\max }^{m}$.

In an effective hyper-path, it usually includes multiple continuous arcs, in which the traveler travels a distance in a certain traffic subnet before transferring to another subnet in the appropriate transfer point. The hyper-path is defines as: a continuous arc sequence between adjacent arcs, transfer arcs, and leaving arcs in an effective hyper-path of a multimodal network. Obviously, the effective super path is composed of the Internet arc, Sub path, transfer arc and lower net arc. The formula (4) is one sub-path of the hyper-path represented by the formula (3):

$$
\left(m_{1}, v_{1}\right)-\left(m_{1}, v_{2}\right)-\ldots-\left(m_{1}, v_{n}\right) \text {. }
$$


Figure 1 shows a multimodal super network, including cars, electric cars, bicycles and bus four sub-nets, can be represented as c, e, b and p.

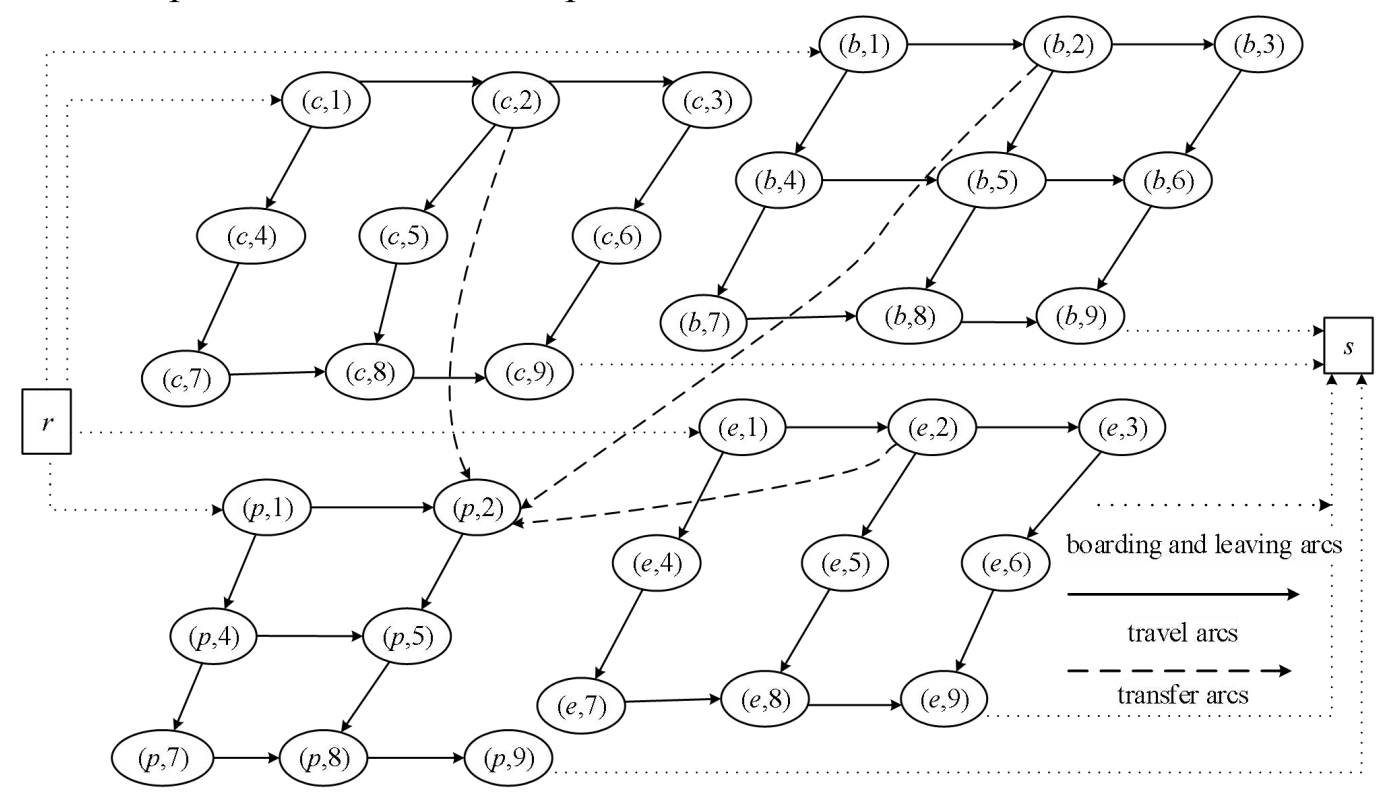

Fig.1 the multimodal Super-network

\section{Generalized costs considering distance factors}

In the multimodal network, the travel choice includes the choice of the mode and the path, and the factors that affect the travel choice include time, cost, transfer and comfort. In the previous study, the influence of distance factor on traveler's travel choice was rarely considered, however, the distance factor was an important factor that affected traveler's travel choice, especially the mode choice. As the distance increases, the cost increases, the comfort decreases, and the sensitivity to distance are different between each mode.

According to the above analysis, travelers' process in the network travel by the boarding arcs, sub-path, transfer arc and the leaving arc to describe, the corresponding travel costs can be used in these arcs of the cost to represent, the following analysis and modeling respectively.

(1)Boarding and leaving cost

The boarding cost indicates the time taken by the traveler to reach the neighboring network nodes from the original of the trip, and the cost of the leaving arc is the time spent by the traveler from the last network node to the destination of the trip. Typically, these two types of costs can be assumed to be constant, i.e.

$$
\begin{array}{ll}
t_{a}=\mu_{r}^{m, v}, & a \in A_{1} . \\
t_{a}=\omega_{m, v}^{s}, & a \in A_{4} .
\end{array}
$$

Where $\mu_{r}^{m, v}$ and $\omega_{m, v}^{s}$ are given constants.

(2) The cost of transfer arcs

Transfer cost refers to transfer time, which consists of walking time and waiting time, and the transfer time is assumed to be a constant. Because the transfer requires physical exertion, therefore, the transfer of the traveler's psychological awareness costs should be moderately enlarged transfer time, can be expressed as:

$$
t_{a}=\eta \cdot h_{m_{1}, v}^{m_{2}, v}, \quad a \in A_{3} .
$$

Where $h_{m_{1}, v}^{m_{2}, v}$ denotes transfer time, $\eta$ is a coefficient factor greater than 1 .

(3) The cost of sub-path

By definition, a sub-path consists of a travel arc of the same category, the cost of which represents the cost of a traveler traveling a distance in a subnet. The factors that affect travelers' travel choices include time, price, comfort and so on, in which the time factor is driving time, price factors 
include fare, fuel consumption and so on, comfort refers to the psychological cost of internal crowding. In a travel, these factors are related to distance.

Firstly, in the mode $m$ of the travel of the arc, travel time can be expressed as the monotonically increasing function of road link flow, if the mutual interference between different modes, the available formula (8) calculation[7]

$$
t_{a}^{m}=t_{a}^{m(0)} \cdot \prod_{m}\left(1+\alpha_{m}\left(\frac{V_{a}^{m}}{C_{a}^{m}}\right)^{\beta_{m}}\right), \quad a \in A_{2}, m \in M .
$$

Where $t_{a}^{m(0)}$ is the free flow travel time, $C_{a}^{m}$ is the capacity of travel arcs, $V_{a}^{m}$ is the flow of different vehicle, $\alpha_{m}$ and $\beta_{m}$ are parameters.

The average number of passengers in different modes is different, therefore, the flow of different modes of travel demand is different, can be used (9) to calculate the flow of different modes ${ }^{[7]}$ :

$$
V_{a}^{m}=x_{a}^{m} \cdot \frac{U^{m}}{Z^{m}}, \quad a \in A_{2}, m \in M .
$$

Where $x_{a}^{m}$ denotes the traffic demand of mode $m, Z^{m}$ denotes the average number of passengers of mode $m, U^{m}$ denotes the Conversion coefficient of passenger car unit.

In addition to time, there are in-car congestion brings comfort costs, especially for the public transportation. Therefore, the travel time needs to be amplified to indicate the impact of comfort on travel, as shown in the formula (10).

$$
c_{a}^{m}=t_{a}^{m} \cdot\left\{1+\theta_{m}\left(\frac{x_{a}^{m}}{C_{\max }^{m}}\right)^{\gamma_{m}}\right\}, \quad a \in A_{2}, m \in M .
$$

Where $c_{a}^{m}$ denotes the travel time costs associated with comfort, $C_{\max }^{m}$ denotes the Maximum number of passengers, $\theta^{m}$ and $\gamma^{m}$ are parameters, $\theta^{m}$ is Car crowding coefficient, it is greater than 0 for bus and equals 0 for other modes.

At the same time, with the increase of distance, the cost of time and comfort will be enlarged gradually, and the sensitivity of different modes to distance is different. Therefore, the travel time and comfort cost on a sub-path $l_{k}$ of a hyper-path $k$ is shown in the formula (11):

$$
\tilde{c}_{l_{k}}^{r s, m}=f\left(d_{l_{k}}^{r s, m}\right) \cdot \sum_{a} c_{a}^{m} \delta_{a, l_{k}}^{r s}, \quad r, s \in V_{1}, m \in M, k \in K_{r s}, l_{k} \in k .
$$

Where $d_{l_{k}}^{r s, m}$ denotes the distance of mode $m$ on the sub-path $l_{k}$ of the effective hyper-path $k$, can be represented by formula (12), $f\left(d_{l_{k}}^{r s, m}\right)$ is a monotone increment function of $d_{l_{k}}^{r s, m}$, can be represented by formula (13), $\delta_{a, l_{k}}^{r s}$ is correlation coefficient, if travel arc $a$ is on the sub-path $l_{k}$ of the effective hyper-path $k, \delta_{a, l_{k}}^{r s}=1$, otherwise $\delta_{a, l_{k}}^{r s}=0$.

$$
d_{l_{k}}^{r s, m}=\sum_{a} d_{a}^{m} \cdot \delta_{a, l_{k}}^{r s}, r, s \in V_{1}, m \in M, k \in K_{r s}, l_{k} \in k .
$$

Where $d_{a}^{m}$ denotes the distance of mode $m$ on the travel $\operatorname{arc} a$.

$$
f\left(d_{l_{k}}^{r s, m}\right)=a^{m} \cdot d_{l_{k}}^{r s, m}+b^{m} .
$$

Where $a^{m}$ and $b^{m}$ are parameters.

The factors that affect the traveler's traffic behavior include the time of the sub-path cost and the psychological perception cost of comfort, and the price factor. Usually, the price cost is directly related to travel distance, the longer the distance, the higher the price cost. The price charge available for a sub-path on an effective hyper-path between OD pairs $r$-s shown in the formula (14):

$$
P_{l_{k}}^{r s, m}=\kappa_{m}+\pi_{m} \cdot d_{l_{k}}^{r s, m}, \quad r, s \in V_{1}, m \in M, k \in K_{r s}, l_{k} \in k .
$$

Where $P_{l_{k}}^{r s, m}$ denotes the price cost of sub-path, $\kappa_{m}$ and $\pi_{m}$ are parameters, $\kappa_{m}$ is the base price, $\pi_{m}$ is the price increase rate.

Therefore, for a sub-path on a $O D$ pair $r-s$ of hyper-path, the generalized cost $g_{l_{k}}^{r s, m}$ is shown in the formula (15): 


$$
g_{l_{k}}^{r s, m}=\tilde{c}_{l_{k}}^{r s, m}+\tau \cdot P_{l_{k}}^{r s, m}
$$

Where $\tau$ is parameter.

From the above analysis, the cost of the super-path is composed of the boarding cost, the sub-path cost, the transfer cost and the leaving cost. For an effective hyper-path between $O D$ pair $r-s$ in a hyper-network, the generalized cost $G_{k}^{r s}$ is shown in the formula (16):

$$
G_{k}^{r s}=\sum_{a \in A_{1}} \mu_{r}^{m, v} \delta_{a, k}^{r s}+\sum_{l_{k} \in k} g_{l_{k}}^{r s, m}+\sum_{a \in A_{3}} t_{a}^{m} \delta_{a, k}^{r s}+\sum_{a \in A_{4}} \omega_{m, v}^{s} \delta_{a, k}^{r s}, \quad r, s \in V_{1}, m \in M, k \in K_{r s}, l_{k} \in k .
$$

where $\delta_{a, k}^{r s}$ is correlation coefficient, if arc a is on the effective hyper-path $\mathrm{k}$ of the $O D$ pair $r-s$, $\delta_{a, k}^{r s}=1$, otherwise $\delta_{a, k}^{r s}=0$.

Only travel arc cost is related to flow, take the link flow as the variable, travel arc time cost can be expressed as formula (17):

$$
c_{a}^{m}\left(x_{a}^{m}\right)=t_{a}^{m(0)} \cdot \prod_{m}\left[1+\alpha_{m}\left(\frac{x_{a}^{m} U^{m}}{C_{a}^{m} Z^{m}}\right)^{\beta_{m}}\right] \cdot\left[1+\theta_{m}\left(\frac{x_{a}^{m}}{C_{\max }^{m}}\right)^{\gamma_{m}}\right] .
$$

The hyper-path generalized cost can be expressed as formula (18):

$$
\begin{aligned}
& G_{k}^{r s}=\sum_{l_{k} \in k}\left[\left(a^{m} \cdot \sum_{a \in A_{2}} d_{a}^{m} \delta_{a \downarrow_{k}}^{r s}+b^{m}\right) \cdot \sum_{a \in A_{2}} c_{a}^{m}\left(x_{a}^{m}\right) \delta_{a l_{k}}^{r s}\right]+\tau \cdot \sum_{l_{k} \in k}\left(\kappa_{m}+\pi_{m} \cdot \sum_{a \in A_{2}} d_{a}^{m} \delta_{a l_{k}}^{r s}\right)+ \\
& \sum_{a \in A_{1}} \mu_{r}^{m, v} \delta_{a, k}^{r s}+\sum_{a \in A_{3}} \eta h_{m_{1}, v}^{m_{2}, v} \delta_{a, k}^{r s}+\sum_{a \in A_{4}} \omega_{m, v}^{s} \delta_{a, k}^{r s}, \quad r, s \in V_{1}, m \in M, k \in K_{r s}, l_{k} \in k .
\end{aligned}
$$

\section{User Equilibrium Assignment Model}

In this paper, we choose user equilibrium criteria for flow assignment. When the flow equilibrium condition is reached, the cost and flow in the effective super-path between $r$ and s of OD pair have the following relationship:

$$
\left\{\begin{array}{l}
\mu_{r s}-G_{k}^{r s}=0, \quad f_{k}^{r s}>0 \\
\mu_{r s}-G_{k}^{r s} \leq 0, \quad f_{k}^{r s}=0 \\
\sum_{r} \sum_{s} \sum_{k} f_{k}^{r s}=q^{r s} \\
r, s \in V_{1}, k \in K_{r s}
\end{array} .\right.
$$

where $\mu_{r s}$ denotes the minimum cost between $\mathrm{r}$ and $\mathrm{s}$ of OD pair under the equilibrium condition; $f_{k}^{r s}$ is the flow of the effective super-path and $q^{r s}$ is the total demand.

The problem can be written as a variational inequality model using the path cost function. The equivalence proof is given in [8]. That is to find the balanced path flow $f_{k}^{r s^{*}} \in \Omega$, Let $\forall f_{k}^{r s} \in \Omega$ satisfy the following condition:

$\sum_{r} \sum_{s} \sum_{k} G_{k}^{r s}\left(f_{k}^{r s^{*}}\right)\left(f_{k}^{r s}-f_{k}^{r s^{*}}\right) \geq 0$.

In this condition,

$$
\Omega=\left\{f_{k}^{r s} \mid x_{a}^{m}=\sum_{r} \sum_{s} \sum_{k} f_{k}^{r s} \delta_{a, k}^{r s}, x_{a}^{m} \geq 0, f_{k}^{r s} \geq 0, \sum_{r} \sum_{s} \sum_{k} f_{k}^{r s}=q^{r s}, r, s \in V_{1}, k \in K_{r s}, a \in A, m \in M\right\} .
$$

\section{Model solution algorithm}

The continuous averaging algorithm (MSA) is used to solve the model [8]. The specific steps are as follows:

Step1: Initialization: According to the initial cost of each arc, the algorithm searches for the shortest effective super-path and allocate "all-have or all-nothing" to get the allocated flow $x_{a}^{m(1)}(\forall a)$ of each arc. Here, set the number of iterations to 1 .

Step2: Calculate the generalized expense of each arc segment and sub-path according to the current traffic volume $x_{a}^{m(n)}(\forall a)$ 
Step3: Search for the shortest super-path according to the cost of the arcs and sub-paths, and perform the "all-have or all-nothing" distribution according to the demand $q^{r s}$ of OD to obtain additional traffic $y_{a}^{m(n)}, \forall a$.

Step4: Iterative update, calculation $x_{a}^{m(n+1)}=x_{a}^{m(n)}+\frac{1}{n}\left(y_{a}^{m(n)}-x_{a}^{m(n)}\right)$.

Step5: Check Convergence. If satisfied $\sqrt{\sum_{a}\left(x_{a}^{m(n+1)}-x_{a}^{m(n)}\right)^{2}} / \sum_{a} x_{a}^{m(n)} \leq \varepsilon$, then the algorithm is over; otherwise, turn to Step2.

In order to solve the above problem, we must first determine the shortest effective super-path. The traditional shortest path algorithm cannot describe the sub-path problem considered in this paper. Therefore, based on the algorithm of Dijkstra, the shortest and effective super-path search algorithm considering sub-path is proposed.

The specific steps are as follows:

First of all, we give the parameters involved in the algorithm: $i$ is the current node, $j$ is the adjacent node of $i, P_{j}$ is the immediate node of $j, P_{j}^{*}$ is the starting point of the sub-path of $j ; L\left(P_{j}^{*}, j\right)$ is the distance from the starting point $P_{j}^{*}$ to $j, G_{j}$ is the minimum cost from starting point $r$ to the node $P_{j}^{*}, G_{P_{i}^{*} j}$ is the cost from the starting point $P_{j}^{*}$ to $j$ of the sub-path, $G_{r j}$ is the cost from the starting point $r$ to $j, n(r, j)$ is the number of transfers from the starting point $r$ to $j . m(i, j)$ is the type of mode $(i, j)$ used by the arc, if the mode has travel distance restrictions, such as electric vehicles, then $m(i, j)=1$ otherwise $m(i, j)=0 ; L_{\max }^{m}$ is the maximum travel distance under the mode of the limited travelable distance. $s(i)$ is the label property of the point $i$, when the algorithm found the shortest path from the starting point $r$ to $i$. Then $s(i)=1$, otherwise $s(i)=0 . C_{i}$ is a collection of points that are adjacent with $i$ and has a label property of $0, K$ is the number of iterations.

Step1: Initialization, assigned to the relevant parameters; set $K=0, i=r, n(r, i)=0(\forall i), L\left(P_{j}^{*}, j\right)=0\left(\forall j, P_{j}^{*}\right), G_{j}=0(\forall j), G_{P_{i}^{*} j}=0(\forall j), G_{r j}=\infty(\forall j \neq r), s(r)=1$, $s(i)=0(\forall i \neq r)$.

Step2: Take a node, such as $j$ :

(1) If the $\operatorname{arc}(i, j) \in A_{1}$, to determine whether the condition $G_{r j}>\mu_{r}^{m, v}$ is true, if it is true, then $G_{r j}=\mu_{r}^{m, v}, P_{j}^{*}=j, P_{\mathrm{j}}=i, G_{r p_{j}^{*}}=\mu_{r}^{m, v}$,otherwise turn step6.

(2) If the arc $(i, j) \in A_{2}$,

a) if $m(i, j)=1$, to determine whether the condition $L\left(P_{i}^{*}, i\right)+d_{a}^{m} \leq L_{\max }^{m}$ is true, if it is true, then turn Step3, otherwise turn Step6.

b ) if $m(i, j)=0$, then turn Step3

(3) If $(i, j) \in A_{3}$, to determine whether the condition $\left(p_{i}, i\right) \in A_{3}$ is true, if it is true, and then turn Step6, otherwise turn Step4.

(4) If $(i, j) \in A_{4}$, to determine whether the condition $G_{r j}>G_{r i}+\omega_{m, v}^{s} P_{\mathrm{j}}=i$ is true, if it is true, and then turn Step6, otherwise turn Step6.

Step3: To determine whether the condition $G_{r j}>G_{j}+G_{P_{*}^{*} j} P_{\mathrm{j}}=i$ is true, if it is true, and then let $G_{r j}=G_{j}+G_{P_{i}^{*} j}, \quad G_{j}=G_{i}, \quad P_{j}^{*}=P_{i}^{*}, \quad P_{\mathrm{j}}=i$ and turn Step6, otherwise turn Step6.

Step4: Let $n(r, j)=n(r, i)+1$ and determine whether the condition $n(r, j) \leq n_{\max }$ is true, if it is true, and then turn Step5, otherwise turn Step6.

Step5: To determine whether the condition $G_{r j}>G_{j}+G_{P_{i}^{*}}+t_{a}^{m}\left(a \in A_{3}\right)$ is true, if it is true, let $G_{r j}=G_{j}+G_{P_{i}^{*} i}+t_{a}^{m}\left(a \in A_{3}\right), G_{r p_{j}^{*}}=G_{j}+G_{P_{i}^{*} i}+t_{a}^{m}\left(a \in A_{3}\right), P_{j}^{*}=j, P_{\mathrm{j}}=i$, and turn Step6, otherwise turn Step6.

Step6: Remove $j$ from $C_{i}$, to determine whether $C_{i}$ is empty, if it is true, then turn to Step7; otherwise turn to Step2; 
Step7: Find the least expensive point from the point labeled 0 , such as $j$, let $s(j)=1$ and set $j$ as the current node, ;let $K=K+1$, then turn Step8;

Step8: If the judgment $s(s)=1$ is true, then the algorithm is over. We should find the shortest cost $G_{r s}^{\min }$ of the effective super-path, and the shortest and effective super-path is found by using the immediately preceding node; otherwise, turn to Step 2.

\section{Example analysis}

We take the network of Figure 1 as an example to verify the model and the algorithm, the values of the parameters are as follows:

$$
\eta=1.100, \quad \varepsilon=0.001, \tau=0.200, \quad n_{\max }=2, \quad L_{\max }^{b}=10 \mathrm{~km}, \quad L_{\max }^{e}=20 \mathrm{~km}, \quad q_{r s}=10000 \text { person }
$$

The information in each mode is shown in table 1 3

Table 1 Relevant Information of Arcs

\begin{tabular}{|c|c|c|c|c|c|c|c|}
\hline travel arcs & $\begin{array}{c}t_{a}^{e(0)}\left(t_{a}^{c(0)}\right) \\
{[\mathrm{h}]}\end{array}$ & $\begin{array}{l}t_{a}^{b(0)} \\
{[\mathrm{h}]}\end{array}$ & $t_{a}^{p(0)}[\mathrm{h}]$ & $C_{a}^{c}\left(C_{a}^{e}\right)[\mathrm{pcu} / \mathrm{h}]$ & $C_{a}^{b}[\mathrm{pcu} / \mathrm{h}]$ & $C_{a}^{p}[\mathrm{pcu} / \mathrm{h}]$ & $\begin{array}{l}L(i, j) \\
{[\mathrm{km}]}\end{array}$ \\
\hline$\{(m, 1),(m, 2)\}$ & 0.067 & 0.133 & 0.100 & 1000 & 600 & 1000 & 2.000 \\
\hline$\{(m, 2),(m, 3)\}$ & 0.167 & 0.300 & 0.250 & 700 & 400 & & 5.000 \\
\hline$\{(m, 1),(m, 4)\}$ & 0.122 & 0.367 & 0.275 & 1500 & 800 & 1500 & 5.500 \\
\hline$\{(m, 2),(m, 5)\}$ & 0.250 & 0.333 & 0.375 & 700 & 400 & 700 & 7.500 \\
\hline$\{(m, 3),(m, 6)\}$ & 0.233 & 0.400 & & 700 & 400 & & 7.000 \\
\hline$\{(m, 4),(m, 5)\}$ & & 0.267 & 0.225 & & 600 & 1000 & 4.500 \\
\hline$\{(m, 5),(m, 6)\}$ & & 0.267 & & & 600 & & 5.000 \\
\hline$\{(m, 4),(m, 7)\}$ & 0.111 & 0.367 & 0.238 & 900 & 500 & 900 & 5.000 \\
\hline$\{(m, 5),(m, 8)\}$ & 0.150 & 0.333 & 0.225 & 700 & 400 & 700 & 6.000 \\
\hline$\{(m, 6),(m, 9)\}$ & 0.167 & 0.333 & & 700 & 400 & & 5.000 \\
\hline$\{(m, 7),(m, 8)\}$ & 0.111 & 0.333 & 0.237 & 900 & 500 & 900 & 5.000 \\
\hline$\{(m, 8),(m, 9)\}$ & 0.167 & 0.333 & 0.250 & 900 & 500 & 900 & 5.000 \\
\hline
\end{tabular}

Table 2 Information of Other Links

\begin{tabular}{|c|c|c|}
\hline $\operatorname{arcs}$ & category & Time[h] \\
\hline$\{r,(c, 1)\}$ & $\begin{array}{c}\text { boarding } \\
\text { arc }\end{array}$ & 0.050 \\
\hline$\{r,(b, 1)\}$ & $\begin{array}{l}\text { boarding } \\
\text { arc }\end{array}$ & 0.033 \\
\hline$\{r,(p, 1)\}$ & $\begin{array}{l}\text { boarding } \\
\text { arc }\end{array}$ & 0.117 \\
\hline$\{r,(e, 1)\}$ & $\begin{array}{l}\text { boarding } \\
\text { arc }\end{array}$ & 0.050 \\
\hline$\{(c, 2),(p, 2)\}$ & transfer arc & 0.083 \\
\hline$\{(b, 2),(p, 2)\}$ & transfer arc & 0.033 \\
\hline$\{(e, 2),(p, 2)\}$ & transfer arc & 0.083 \\
\hline$\{(c, 4), s\}$ & leaving arc & 0.050 \\
\hline$\{(b, 4), s\}$ & leaving arc & 0.033 \\
\hline$\{(p, 4), s\}$ & leaving arc & 0.117 \\
\hline$\{(e, 4), s\}$ & leaving arc & 0.050 \\
\hline
\end{tabular}


Table 3 Information of Different Traffic Modes

\begin{tabular}{ccccc}
\hline $\begin{array}{c}\text { mode } \\
\text { parameter }\end{array}$ & $c$ & $b$ & $e$ & $p$ \\
\hline$Z^{m}$ & 4 & 1 & 4 & 20 \\
$U^{m}$ & 1 & 0.250 & 1 & 1.500 \\
$C_{\max }^{m}$ & 4 & 1 & 4 & 40 \\
$\alpha^{m}$ & 0.150 & 0.150 & 0.150 & 0.150 \\
$\beta^{m}$ & 4 & 4 & 4 & 4 \\
$\theta^{m}$ & 0 & 0 & 0 & 0.150 \\
$\gamma^{m}$ & 4 & 4 & 4 & 4 \\
$a^{m}$ & 0.005 & 0.100 & 0.005 & 0.010 \\
$b^{m}$ & 1 & 1 & 1 & 1 \\
$\kappa_{m}$ & 2 & 0 & 2 & 1 \\
$\pi_{m}$ & 0.200 & 0 & 0.150 & 0.060 \\
\hline
\end{tabular}

Firstly, the convergence of the algorithm is analyzed, and the convergence is represented by the flow change of the car and the electric vehicle in each iteration. Figure 2 shows the first 30 calculations of the MSA algorithm. It can be seen that the algorithm has obvious convergence property.

There are 5 effective super-paths in balance condition, as shown in table 4, the effective hyper -path of the bike and the electric vehicle sub-path distance is not over the distance limit, the path exceeding the distance limit is not selected, such as path $r-(e, 1)-(e, 4)-(e, 7)-(e, 8)-(e, 9)-s$. Figure 3 shows the traffic demands of each arc, bicycle flow is only distributed on the arc $\{(b, 1),(b, 2)\}$, because the bicycle mode is only suitable for short distance travel, only the hyper-path of $r-(b, 1)-(b, 2)-(p, 2)-(p, 5)-(p, 8)-(p, 9)-s$ the bicycle path is not more than $10 \mathrm{~km}$, the rest of the path of the bicycle distance too long.

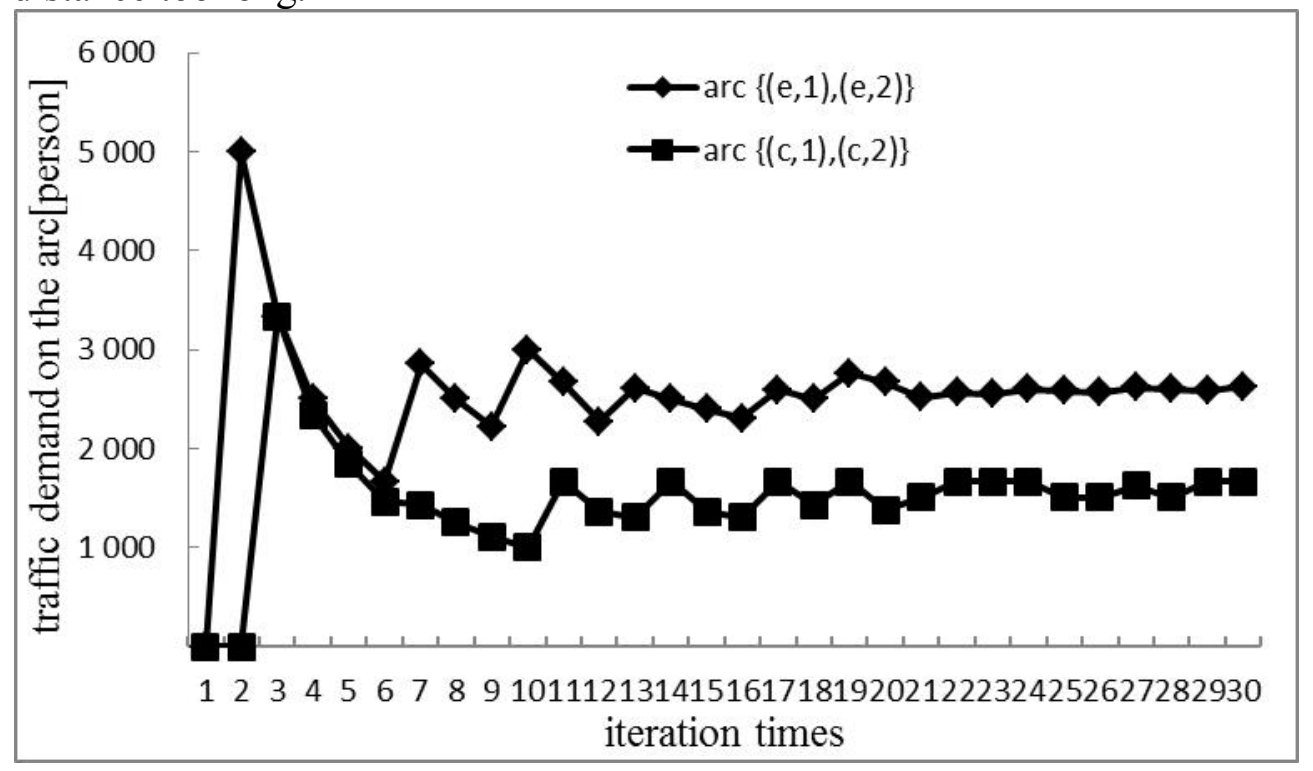

Fig.2 The convergence of algorithm 
Table 4 Assignment results of effective hyper-route

\begin{tabular}{|c|c|c|c|c|c|}
\hline $\begin{array}{l}\text { Path } \\
\text { number }\end{array}$ & Hyper-path & $\begin{array}{l}\text { Transfer } \\
\text { times }\end{array}$ & $\begin{array}{l}\text { Sub-path } \\
\text { distance } \\
\text { and mode }\end{array}$ & $\begin{array}{l}\text { Sub-path } \\
\text { and mode }\end{array}$ & $\begin{array}{l}\text { Hyper-path } \\
\text { cost }\end{array}$ \\
\hline 1 & $\begin{array}{c}r-(c, 1)-(c, 2)-(c, 5)- \\
(c, 8)-(c, 9)-s\end{array}$ & 0 & $20.500(c)$ & $2.221(c)$ & 2.321 \\
\hline 2 & $\begin{array}{c}r-(c, 1)-(c, 4)-(c, 7)- \\
(c, 8)-(c, 9)-s\end{array}$ & 0 & $20.500(c)$ & $2.248(c)$ & 2.348 \\
\hline 3 & $\begin{array}{c}r-(b, 1)-(b, 2)-(p, 2)- \\
(p, 5)-(p, 8)-(p, 9)-s\end{array}$ & 1 & $\begin{array}{c}2.000(b) \\
18.500(p)\end{array}$ & $\begin{array}{l}0.204(b) \\
2.012(p)\end{array}$ & 2.399 \\
\hline 4 & $\begin{array}{c}r-(p, 1)-(p, 4)-(p, 5)- \\
(p, 8)-(p, 9)-s\end{array}$ & 0 & $21.000(p)$ & $2.162(p)$ & 2.396 \\
\hline 5 & $\begin{array}{c}r-(e, 1)-(e, 2)-(e, 3)- \\
(e, 6)-(e, 9)-s \\
\end{array}$ & 0 & $19.000(e)$ & $2.203(e)$ & 2.303 \\
\hline$r$ & $\underbrace{3167}_{167}$ & 7 & & 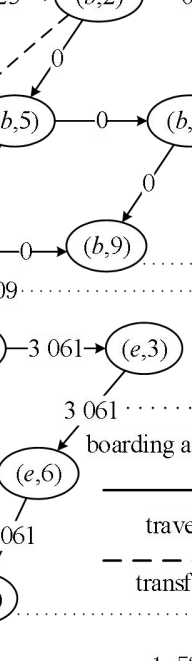 & 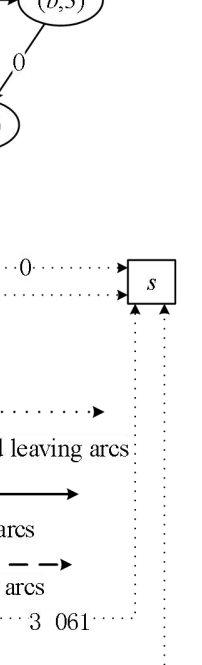 \\
\hline
\end{tabular}

Fig. 3 The link flow of super network at equilibrium

\section{Conclusion}

Based on the graph theory, a super-network model for describing the multimodal combined travel is established, and defines the effective hyper-path and sub-path. Then, the travel cost of travelers in different travel stages is analyzed, the influence of distance factor on time and price is considered, and the generalized cost model of multimodal combination travel is constructed. On this basis, a multimodal traffic network assignment model based on user equilibrium criterion and an efficient shortest path search algorithm are proposed. Finally, an example is used to verify the model and the algorithm, the result shows that the proposed model and algorithm are effective, and the travel distance is an important factor that affects the traveler's mode selection and route selection.

\section{References}

[1] B.F. Si, M. ZHONG, X.B. Yang, Z.Y. Gao. Urban transit assignment model based on augmented network with in-vehicle congestion and transfer congestion[J]. Journal of Systems Science and Systems Engineering, 2011, 20(2): 155-172.

[2] J.F. Liu. Transfer-based modeling flow assignment with empirical analysis for urban rail transit network[D]. Beijing: Beijing Jiaotong University, 2012. 
[3] B.F. Si, X.B. Yang, L. Gao, et al. Urban multimodal traffic assignment model based on travel demand[J]. China Journal of Highway and Transport, 2010, 23(6): 85-91.

[4] Q.Z. Wang, B.F. Si. Urban multi-modal traffic assignment model and algorithm under transfer constrain[J]. Journal of Transportation Systems Engineering and Information Technology, 2017, 17(4): 159-165.

[5] J.E. Kang, W.W. Recker. An activity-based assessment of the potential impacts of plug-in hybrid electric vehicles on energy and emissions using 1-day travel data[J]. Transportation Research Part D Transport \& Environment, 2009, 14(8): 541-556.

[6] F. He, Y.F. Yin, LAWPHONGPANICH S. Network equilibrium models with battery electric vehicles[J]. Transportation Research Part B Methodological, 2014, 67(3): 306-319.

[7] B.F Si, M. Zhong, Z.Y. Gao. A link resistance function of urban mixed traffic network[J]. Journal of Transportation Systems Engineering and Information Technology, 2008, 8(1): 68-73.

[8] B.F. Si, Z.Y. Gao. Modeling network flow and system optimization for traffic and transportation system[M]. Beijing: China Communications Press, 2013. 